\title{
Premature ventricular complexes (PVC)-induced cardiomyopathy: A review and meta-analysis of pathophysiology, diagnosis and clinical management
}

\author{
Aref Albakri* \\ St-Marien hospital Bonn Venusberg, department of Internal Medicine, Bonn, Germany
}

\begin{abstract}
Frequent premature ventricular complexes (PVC) is a common cardiac entity in patients with structural heart disease and PVC-induced cardiomyopathy (PICM). It is also common in asymptomatic patients, where it is considered a benign entity with no prognostic implications. In patients with structural heart disease, the presence of PVCs can result in fatal ventricular arrhythmias. Various cellular and extracellular mechanism as well as risk factors for developing PICM have been suggested but the exact mechanisms remains unclear. The increasing insights into the treatment of ventricular tachyarrhythmias, especially the introduction of radiofrequency catheter ablation in clinical practice has renewed interest in PVCs as predictors of arrhythmias as well as their potential aetiological association with CM. The present paper reviews existing evidence on PICM patients with a focus on prevalence, risk factors, pathophysiology, clinical presentation, concluding with and a meta-analysis and discussion of current diagnostic and treatment criteria.
\end{abstract}

\section{Introduction}

Premature ventricular contractions (PVCs: also known as premature ventricular complexes, ventricular extrasystoles or ventricular premature beats) are a common entity co-existing with cardiomyopathy (CM) and other structural heart diseases yet their effects on the cardiovascular system are not entirely clear. Their prognostic significance is interpreted after considering the presence or absence of an underlying structural heart disease. In the absence of structural heart disease, PVCs are a benign cardiac entity although these studies enrolled a small sample and performed fewer cardiac tests on follow-up $[1,2]$. In the presence of structural heart disease, PVCs may carry adverse prognosis. In the 1970s and 1980s, frequent PVCs were postulated to be a trigger for ventricular tachycardia (VT), ventricular fibrillation (VF) and sudden cardiac death (SCD) in post myocardial infarction (MI) patients and PVCs suppression was thought to be warranted in this context [3]. Case reports [4-6] and a small observational and non-randomized study [7] in the late 1990s and early 2000s were the first to report improvement or resolution of CM after the elimination of PVCs. These observations led to the description of a clinical cardiac entity described as PVC-induced CM (PICM). Although PICM has been demonstrated in canines [8], in humans it is inferred due to observed reversal of LV dysfunction upon successful suppression of PVCs or from the presence of PVCs in a structurally normal heart and subsequent LV dysfunction. However, the most current classification by the American Heart Association (AHA) classifies PICM as one of the secondary aetiologies of DCM [9] while the ESC does not specifically mention PICM [10]. The current clinical conundrum of comorbid PVC and CM is to recognize when PVCs are the cause or the consequence of CM. The purpose of the present review is to discuss what is known about PICM, its pathophysiology, diagnosis and clinical management.

\section{Definition of PICM}

Cardiac arrhythmias are an abnormal heart rhythm. They result from any disruption in the regularity, rate or transmission of cardiac electrical impulse. Among the various abnormalities, PVC is among the most significant arrhythmias due to ectopic heartbeats. It is a widespread form of arrhythmia in adults with or without underlying structural heart disease. At the cellular level, myocytes in the ventricular myocardial spontaneously depolarize early to create an extra systole (heartbeat), which is disharmonious with the cardiac cycle. These extra beats disrupt the regular cardiac rhythm potentially causing the perception of a flutter or a skipped beat. PVCs are the most common ventricular arrhythmias. Frequent PVCs can result in electromechanical dyssynchrony leading to the development of a specific type of CM potentially reversible by the therapeutic suppression of PVCs.

\section{Prevalence and prognosis}

The prevalence of PVCs depends on the comorbidities of the screened patients and the duration of monitoring. In the general population, the prevalence of PVCs (those having $>60 \mathrm{PVCs} / \mathrm{h}$ ) ranges between $1 \%$ and $4 \%$ [11]. In a normal healthy population, PVCs occur in about $1 \%$ on standard 12-lead ECG and raises to between $40 \%$ and $75 \%$ on 48 -hour Holter monitoring [12]. The prevalence of PVCs shows an age dependent pattern with a highly disproportionate burden on the geriatric population [13]. In children younger than 11 years, the

${ }^{*}$ Correspondence to: Aref Albakri, St-Marien hospital Bonn Venusberg, department of Internal Medicine, Bonn, Germany, E-mail: arefalbakri@yahoo.com

Key words: ablation, premature ventricular complex (PVC)-induced cardiomyopathy (PICM), dilated cardiomyopathy (DCM), PVC burden

Received: September 05, 2019; Accepted: September 30, 2019; Published: October 05, 2019 
prevalence of PVCs is less than $1 \%$ [14] rising to $69 \%$ in older adults ( 75 years of age) [15]. However, the current prevalence of PVCs in the general population and its age distribution remains unknown since the cited prevalence relies on studies performed in the early 1980s [1315]. Thus, there is need for population-based epidemiological studies to clarify the current prevalence of PVCs in the general population as well as in patients with co-existing structural heart diseases including its geographical distribution.

The prognostication of PVCs in the general population and in patients with co-occurring $\mathrm{CM}$ and other structural heart diseases is an ongoing research area. The seminal mention of the concept of PICM in published medical literature was in 1998 by Duffee and co-workers. [7]. They observed 10 patients with idiopathic dilated CM (DCM) and 4 patients with ischemic heart disease who showed significant improvement in clinical functional status and LV systolic function following successful medical suppression of PVCs. The study postulated the involvement of PVCs in the pathogenesis of a specific type of CM reversible by medical suppression of PVCs. This seminal study inspired subsequent research on the role of PVCs in patients with DCM and other non-ischemic forms of CM. Four subsequent studies confirmed that the suppression of PVCs improved or resolved CM, leading to the recognition of the entity of PICM [4-7]. However, for years since the Duffee study [7], the consideration of isolated PVCs (with no underlying cardiac disease) as a benign entity with no prognostic implications persisted. This conclusion relied on findings of studies with a small sample size and limited diagnostic testing [16]. However, recent studies have demonstrated the potential detrimental effect of frequent PVCs in patients with structurally normal hearts and the development and reversibility of PVC-induced CM $[17,18]$. Frequent PVCs have can also worsen a pre-existing $\mathrm{CM}$, in which case, PVC suppression may only lead to a partial recovery of the LV function [19].

Despite the increasing recognition of PICM both in scholarly discourse and in clinical practice, its exact prevalence both in the general population and in patients with structural heart diseases remain unknown. The possible underlying reason may be PVC is an underappreciated aetiology of LV dysfunction and it is primarily a geriatric condition (frequently observed in polder patients). Underlying this reason is that the prevalence of PVCs correlates with advancement in age or the possibility that PICM develops in a time dependent fashion [17]. Niwano et al. [20] demonstrated progressive worsening of LV function in patients with frequent PVCs (> 1,000 beats per day) as measured by the LVEF and LV end-diastolic dimension (LVEDD) over a follow-up duration of 4 to 8 years. This finding suggests that although the prognosis of patients with frequent PVCs is relatively benign, clinicians should pay attention to the progression of LV dysfunction during a long-term observation especially in patients with a high PVC prevalence.

\section{Risk factors}

Not all patients with PVCs will develop PICM. Some patients with high PVC burden remain asymptomatic and never develop LV dysfunction. Risk factors that may influence the development of PICM are (i) PVC burden; (ii) PVC origin; (iii) QRS features, interpolation and coupling intervals; (iv) male sex; and (v) circadian variability.

\section{PVC burden}

PVC burden is the strongest independent predictor of PICM. A positive correlation between PVC burden, and the sensitivity and specificity to predict the occurrence of PICM has recently emerged [8].
This correlation is attributable to a demonstrated association between the frequency of PVCs, and the extent of LV dysfunction and ventricular dilatation at the time of initial clinical presentation [17,21-24]. Patients with depressed LVEF have a higher mean PVC burden (29\% to 37\%) than patients with preserved LV function (8\% to $13 \%$ ) [18,22,25]. PVC burden is one of the strongest predictor of PVC but a clear cut-off point that should mark the frequency at which $\mathrm{CM}$ is unavoidable remains unclear [26,27]. Current studies provide inconsistent cut-off values [18,25,28]. Ban et al. [28] examined 127 patients with symptomatic PVCs and LV dysfunction but without structural heart disease and reported a PVC burden $>26 \%$ and the presence of retrograde $\mathrm{P}$ waves were independent predictors of PICM. The cut-off point of $>26 \%$ had a sensitivity of $70 \%$ and specificity of $78 \%$ determined by receiver-operator characteristics (ROC) curve analysis. Baman et al. [18] reported cut-off values of $10 \%$ as the minimum PVC burden that appears to result in CM and $>24 \%$ as PVC burden independently associated with PICM with a sensitivity (79\%) and specificity (78\%) in separating patient population with impaired versus preserved LV function. Hasdemir et al. [25] reported a PVC-burden of $\geq 16 \%$ with a sensitivity $90 \%$ and specificity $85 \%$. The variability in the reports cut-off values for PVC-burden among studies may result from patient selection bias and symptomatic status of the patients [27]. It may also result from differences in the frequency of beats to define PVC burden - 20,000 PVCs over 24 hours [20],10,000 per day [24] or $>10 \%$ of total beats instead of the absolute number of PVCs $[18,25]$.

\section{PVC origin}

PVC can originate from the RV or the LV and point of origin may have variable influence on the development of PICM. The anatomic source of two thirds of idiopathic PVCs is the ventricular outflow tract musculature, primarily from the right ventricular outflow tract (RVOT). These include PVCs arising from myocardial extension above the aortic and pulmonary valves (cusps). In an autopsy of 603 hearts, Gami et al. [29] observed 57\% had myocardial extension above the aortic valve and $74 \%$ above the pulmonary valve. About half of the extension (55\%) were more often located in the right coronary cusp than in the left coronary cusp (24\%) and non-coronary or posterior cusp $(<1 \%)$. Myocardial extensions above the right and left coronary, and non-coronary pulmonary cusps have a more even distribution (45 to 60\%), which constitute the electrical substrates for ectopies and tachycardia [29]. The remaining a third of PVCs originate from different ventricular locations (the septum, papillary muscles, free wall or LV fascicles) [30]. They may be from a single or multiple foci presenting as monomorphic or polymorphic on ECG whereas PVCs from RVOT are often monomorphic [26].

The RVOT as a source of PVCs has received extensive research relative to LVOT. It is a common source of VT in patients with a structurally normal heart, usually accompanied by LBBB morphology and inferior axis. Reversible tachycardia-induced CM has been associated with RVOT-VT that responds well to RFA with a complete recovery of the LV function $[16,26]$. Individual series and case reports have shown that PVCs originating from RVOT can cause an impairment of the LV function. Most of the reported cases of a successful recovery of the LV function after RCA of PVCs are related to RVOT-PVCs. Besides a high PVCs burden, the site of origin from the RVOT itself may be a predisposing factor to $\mathrm{CM}$ reversing the activation sequence of the ventricular myocardium [26].

Despite increasing recognition of the association between the site of PVC origin (the RV or LV) and the risk of developing PICM, supporting evidence does not demonstrate a clear association. Del 
Carpio et al. [30] performed a retrospective study that enrolled 70 patients who had underwent PVC ablation. There was no significant differences observed in baseline characteristics between patients with reduced LVEF $(<50 \%)$ and those with preserved LVEF $(\geq 50 \%)$ although those with reduced LVEF were more likely to be symptomatic. In addition to a higher PVC burden in patients with reduced LVEF, the study associated PVCs originating from the RV with a significant reduction in LVEF at PVC burden $\geq 10 \%$ and PVC originating from the LV with a significant reduction in LVEF at PVC burden $\geq$ $20 \%$. Interpretation of these findings however should consider the retrospective nature of the study, small sample size and highly selected patient population. Whether delayed LV excitation and contraction from RV PVCs cause greater hemodynamic threat to myocardial function has not been studied. However, several small-scale studies suggest RV pacing induced LV dysfunction [31-36].

\section{PVC: QRS features, interpolation and coupling intervals}

PVC morphology, QRS features, interpolation, and coupling intervals have been suggested as possible predictors of the development of PICM. The RV PVCs exhibit a lower threshold for PVCs burden associated with depressed LVEF compared to LV but PVC morphology (such as LBBB or RBBB pattern) does not appear to affect LVEF [30]. However, PVC morphology may influence PVCs' site and aetiology. PVCs with smooth uninterrupted contours and sharp QRS deflection suggest an isolated ectopic focus and the absence of structural heart disease. PVCs with a broad notching and slurred QRS deflection suggest a diseased myocardial substrate [26]. A PVC QRS duration $\geq 140 \mathrm{~ms}$ has been reported as an independent predictor of LVEF dysfunction more commonly observed in PVCs originating from the free ventricular wall and outflow tracts $[30,37,38]$. A narrower QRS typically originate from the septum or fascicles. The presence of interpolated PVCs have also been reported as predictive of PICM. A single-centre, small-scale studies associated both interpolated PVCs and PVC burden with a higher risk of PICM [39]. PVC coupling interval $\leq 600 \mathrm{~ms}$ are associated with a lower mean LVEF, possibly due to an abnormal filling of the LV and decreased stroke volume [40,41]. A coupling interval variability of $60 \mathrm{~ms}$ is more frequent in PVCs originating from the sinus of Valsalva or the great cardiac vein associated with increased frequency of cardiac events [42]. Most of the current evidence associating PVCs' QRS duration, interpolation and coupling interval with the development PICM may not apply to all patients because they are single studies with a small number of patients. Thus, larger prospective studies are warranted to confirm the contribution of these PVCs' characteristics, and the development of PICM.

\section{PVC duration}

Besides PVC burden, the duration of symptoms or PVC QRS duration is increasingly emerging as an independent strong predictor of PICM. Yokokawa et al. [37] retrospective analysis of 241 patients who underwent ablation therapy for frequent PVCs. The study observed patients with PICM and depressed systolic function (LVEF $<50 \%)$ have a significantly higher PVC burden (28\% vs. $15 \%)$ and a significantly lower duration of symptoms (135 vs. 35 months) compared with patients with PVCs with preserved LV systolic function (LVEF $>50 \%$ ). In a multivariate analysis, symptom duration of 30 to 60 months, absence of symptoms, and PVC burden in asymptomatic patients were independent predictors of impaired LV function. Since asymptomatic patients are at risk for delayed diagnosis and for the lack of knowledge of the exact time of onset of PVCs, they should be followed frequently by regular echocardiograms for the early detection of LV dysfunction. The observation of a cohort of asymptomatic patients at risk of PICM contradicts the previous belief of the benign nature of PVCs in asymptomatic individuals. Since the Yokokawa et al. [37] study is limited by a retrospective study design, a small sample and non-randomized nature, larger prospective trials with a longer followup may provide clarity in the role of PVC duration and PICM in both asymptomatic and symptomatic patients.

\section{Circadian variability}

Circadian rhythm is a 24-hour cycle in the physiological processes of living beings. In patients with PVCs, circadian variations refers to changes in the PVC frequency during the day, which has been associated with the development of PICM. The consistency in PVC burden throughout the day has been reported as an independent predictor of PICM. Bas et al. [43] performed a prospective study of 107 consecutive patients with mean preserved LVEF (50.4\%) referred for catheter ablation. PICM patients had less variability in circadian PVC distribution (hourly coefficient of variation $31.5 \%$ vs. $59.8 \%$ ) compared to patients without CM. The study concluded that in patients with frequent PVC, consistency in hourly PVC frequency throughout the day is an independent predictor of PICM [43]. In a related study, Hamon et al. [44] enrolled patients with frequent monomorphic PVCs referred for radiofrequency ablation (RFA) suggested that PVC circadian variation may help to predict PVC inducibility in the electrophysiology lab, facilitation the success of RFA procedure. Indeed, patients with a fast heart rate dependent PVCs had the highest successful outcome from RFA. These patients respond to isoproterenol during the procedure. Patients with no correlation between PVCs and mean heart rate had the least successful outcomes [44].

\section{Male sex}

Although PICM disproportionately affects the male gender, supporting evidence is inconsistent on the role of gender in the development of PICM. Two retrospective cohort studies reported the male sex as an independent risk factor for the development of PICM $[45,46]$. Humphries et al. [47] reported a greater incidence of symptomatic PVCs in women and a similar incidence of idiopathic VT in both sexes. Since asymptomatic status could delay the diagnosis and hence facilitate the development of PICM [48], females are less prone to develop PICM because they will be treated much earlier. This sex-related variation may partly be due to hormonal differences but may also be related to differences in symptom perception - females may be more sensitive to PVCs and seek medical attention sooner than males [49].

\section{Mechanism and pathophysiology}

The exact mechanisms underlying the development of PICM is not entirely clear. However, several postulations exist that attempt to explain the pathophysiology of PICM. These mechanisms include tachycardiainduced mechanism, electrochemical dyssynchrony, extrasystolic potentiation, interpolation, R-R variability, and myocardium remodelling from short-coupled PVCs [50]. However, these mechanisms have confounding factors suggesting pathophysiology of PICM is either multifactorial or involves the contribution of other unknown factors.

\section{Tachycardia-induced mechanism}

Tachycardia-induced mechanism is the most commonly hypothesized mechanism, to the extent some researcher considered PICM as a form of tachycardia-induced CM (TIC), which by itself is an established clinical entity [12]. Sufficient evidence exist to support the elimination of tachycardia leads to improvement in the LV function 
in PCIM patients. Sustained tachycardia due to any cause often leads to a state of myocardial stunning by various alterations in the cellular subcellular level such as $\mathrm{Na}^{+}, \mathrm{K}^{+}$, ATPase activity; action potential, myocardial calcium handling, receptor downregulation and oxidative stress [51,52]. However, TIC-mediated mechanisms may not be entirely valid since the improvement in LVEF after the elimination of tachycardia, there is no observable accompanied improvement in other key structural echocardiographic parameters. LV end-systolic and diastolic dimensions may persist for a longer period due to persistent negative remodelling from increased neurohormonal activity and receptor downregulation [53]. In addition, patients with frequent PVCs have overall higher rates similar to those of their normal counterparts on Holter monitoring [20,21].

\section{Electromechanical dyssynchrony}

Electromechanical dyssynchrony is another potential mechanism associated with the development of PICM. Electromechanical dyssynchrony results in a sequential rather than normal parallel excitation of the myocardium. Pacemaker trials associate chronic RV pacing with subsequent impairment in LV function [50]. PVCs especially with LBBB morphology that cause dyssynchronous contraction of the septum and the LV posterior wall can lead to impaired LVEF based on similar electromechanical phenomenon. Huizar et al. [8] performed a randomized animal model study on induced PVC by pacing in mongrel dogs over a period of 12 weeks. At the end of the study period, paced dogs had a significant deterioration of LV function (39.7\% vs. $60.7 \%$ ) compared with the control group. The LV function recovered completely over 2-4 weeks period after stopping the pacing. However, an important finding is that the paced dogs lacked the typical histological features of CM including inflammation, fibrosis, apoptosis and mitochondrial abnormalities.

\section{Other mechanisms}

PVC QRS duration is one of the most independent predictor for the development of PICM. This observation supports the concept that PVC QRS duration reflect subtle underlying myocardial substrate abnormalities that may result in LV dysfunction. Indeed, PVC QRS duration has been used as an accurate measure of the degree of cell-tocell electrical uncoupling, which precedes the development of CM [54]. Deyell et al. [55] proposed that PVC QRS duration is a manifestation of the extent of myofibril disarray and myocardial fibrosis, which are common findings in idiopathic CM. The study also associated PVC QRS duration at the time of ablation with reversibility of PICM. A wider PVC QRS duration may lead to increased myocardial substrate abnormalities and a lower possibility for recovery of CM postablation. Ventricular remodelling may be due to a repetitive abnormal electromechanical activation patter in the context of subtle abnormal myocardial substrates as demonstrated by a wider PVC QRS duration and histopathological and cardiac magnetic resonance imaging (MRI) that could predispose certain patients to develop CM. Interpolated PVCs are also associated with CM independent of increasing PVC burden. Interpolated PVCs have a longer ventriculoatrial block cyclelength compared with PVC without interpolation [39]. The exact mechanism by which interpolation produces $\mathrm{CM}$ is not known.

\section{Clinical presentation}

\section{PVCs}

The most common presenting sign of PVC is sensation of a skipped heartbeat followed by a fluttering sensation. Patients with
PVC commonly complain of heart palpitations. A greater majority of patients are entirely asymptomatic as there are no associated symptoms with the palpitations. However, some patients may experience lightheadedness, chest pain, chest discomfort, dyspnoea and anxiety [24]. Rarely, PVC patients experience syncope due to PVCs. A through history should be include any associated symptom with palpitations, patients medical history, medication including any illicit drug use and supplement wastage [4-6]. Physical examination may reveal irregular heart beat upon auscultation if the patient is experiencing PVCs during examination. Otherwise, there are no direct physical examination findings [9].

\section{PICM}

PICM patients present in one of the three categories: (i) asymptomatic; (ii) symptomatic acutely from individual PVCs; or (iii) symptomatic due to cumulative hemodynamic effects of the PVCs [16]. Asymptomatic patients usually present following incidental discovery on routine ECG or on physical examination. Presentation in asymptomatic patients is often late and asymptomatic status is a risk factor for the development of PICM [37]. Symptoms due to acute effects from individual PVCs may include palpitation, chest discomfort or shortness of breath. Symptoms secondary to the actual PVCs or hyper-contractile beats may succeed compensatory pauses that often follow PVCs. Symptoms due to cumulative effect of frequent PVCs can range from mild fatigue to decompensated HF requiring aggressive therapy. Rarely PICM patients may present with SCD and the presence of frequent PVCs should prompt thorough investigation for occult structural heart disease [16]. Another common presenting feature in patients with an existing bi-ventricular pacing device in whom frequent PVCs can lead to sub-optimal resynchronization benefit. In these patients, the role of PVCs in the initial development of PICM should be considered. Indications to begin therapeutic suppression of PVCs include persistence symptoms, the presence of CM (decreased LVEF or LV dilatation), PVCs inducing malignant ventricular arrhythmias and PVCs limiting optimal bi-ventricular pacing. In asymptomatic patients with a high PVC burden ( $>20 \%$ ), the decision to treat with either medication or ablation to prevent future risk of $\mathrm{CM}$ versus repeat assessment of LV function remains undetermined [16].

\section{Diagnosis and management: A meta-analysis}

PVCs are a common cause of palpitations often diagnosed incidentally on ECG, ambulatory monitoring or inpatient telemetry. They result from early depolarization of ventricular myocytes to create an extra systole uncoordinated with the cardiac cycle. PVCs occur more frequently in patients with structural heart disease, where they are associated with increased risk of HF events including CM. In normal hearts, PVCs are relatively benign although a higher frequency may increase the risk of developing LV dysfunction and subsequently $\mathrm{CM}$. Early diagnosis is essential to improve the success of therapeutic suppression of PVCs and associated reversal of LV dysfunction and even restoration of normal LV function. The pathognomonic feature of PICM is a combination of a high frequency (burden) and evidence of LV systolic dysfunction in the absence of underlying structural heart disease. However, with guidelines on the exact PVC burden lacking, different studies have used difference PVC burden and frequency to define PICM. In addition, data on the efficacy of ablation of PVCs on LV function in asymptomatic or symptomatic patients, and those with or without structural heart diseases are also inconsistent. This meta-analysis pools published data on PICM to determine common diagnostic features and efficacy of ablation therapy on different PICM 
population cohort - symptomatic, asymptomatic, and those with or without structural heart disease.

A systematic online search was performed to identify and select studies that evaluated the diagnosis and efficacy of therapeutic ablation of PVCs in PICM patients. The database consulted in the search were PubMed, Embase and Google Scholar. The key words used in the search were ventricular complexes OR premature ventricular contractions OR ventricular premature beats AND LV dysfunction OR cardiomyopathy AND ablation. Studies included were retrospective and prospective cohort comparing PICM patients with a control group or the same cohort with changes in baseline and post-ablation values. Studies excluded investigated animals, case reports, conference papers and review articles. Data extracted from the studies included study characteristics (first author, study design, publication year, objective and duration of follow-up), patient characteristics (total number recruited, mean age and gender representation) and study outcomes (diagnostic features and therapeutic outcomes). The primary outcome is diagnostic criteria and ablation therapy outcomes on PVC burden and LV function (Table 1).

\section{Findings}

Study characteristics: The electronic search yielded 201 studies. Of the 201, only 11 studies that met the inclusion criteria formed the final dataset for analysis [19-21,45,48,56-61]. These studies are relatively recent, published between 2005 [21] and 2018 [61]. Except one prospective study [58], the remaining 10 studies adopted a retrospective design [19-21,45,48,56,57,59-61]. Two studies [20,21] grouped patients based on PVC burden; three other studies [58,60,61] grouped patients into two - those with and those without structural heart diseases, while the remaining six studies compared PICM patients with LV dysfunction) and control (those with PVC but without LV dysfunction) [19,45,48,56,57,59]. The different objectives of the 11 studies can be subsumed under just two broad objectives: (i) to assess eth efficacy of ablation therapy on LV dysfunction and PVC burden; and (ii) to determine the predictors of PICM on patients with frequent PVCs. In total, the 11 studies enrolled 2,242 patients with frequent PVCs with and without LV dysfunction. The enrolled patients have an equal gender representation (male $=50.1 \%$; female $=49.9 \pm 6.9 \%$ ). The mean follow-up period for the 11 studies to assess the efficacy of ablation of PVCs on LV function and PVC burden on PICM patients was $25.5 \pm 29.4$ months.

Study outcomes: Primary study outcome was to determine common diagnostic criterion for PICM patients, predictors of PICM development and efficacy of ablation therapy on restoration or reversal of LV dysfunction. Diagnosis of PICM patients could not be directly obtained from pooled analysis because of the lack of quantifiable data provided on diagnostic features. Instead, diagnosis was inferred from the inclusion criteria adopted by the individual studies. Common diagnostic methods for PICM is ECG and non-invasive, mostly by echocardiography. PICM patients usually exhibit LV dysfunction or are asymptomatic. Two key diagnostic criterion from the 11 studies were:

1) A high PVC burden $>10 \%$ or $20 \%$ or PVC frequency $>10,000$ to 20,000 beats/day or by 24 hour Holter monitoring;

2) Severe LV systolic dysfunction (LVEF $<50 \%$ ) or LV dilatation by thoracic echocardiography

Table 1. Summary of characteristics of included studies

\begin{tabular}{|c|c|c|c|c|c|c|c|}
\hline $1^{\text {st }}$ Author [Ref \#] & Year & No. & $\begin{array}{c}\text { Male } \\
(\%)\end{array}$ & $\begin{array}{c}\text { Mean } \\
\text { Age }\end{array}$ & Inclusion Criteria & Primary Objective & Main Outcomes \\
\hline Takemoto [21] & 2005 & 28 & 22 & $50 \pm 2$ & $\begin{array}{l}\text { RVOT-PVC; no evidence of } \\
\text { SHD; underwent RFA }\end{array}$ & $\begin{array}{l}\text { To evaluate clinical benefits of } \\
\text { RFA for RVOT-PVC without SHD }\end{array}$ & $\begin{array}{l}\text { RVOT-PVC ( }>20 \%) \text { may cause LV dysfunction and/ } \\
\text { or HF; RFA produces clinical benefits }\end{array}$ \\
\hline Niwano [20] & 2009 & 239 & 49 & $43 \pm 13$ & $\begin{array}{l}\text { Frequent PVCs ( }>1000 \text { beats/ } \\
\text { day) originating from the RVOT/ } \\
\text { LVOT; no SHD }\end{array}$ & $\begin{array}{l}\text { To clarify the prognostic value of } \\
\text { frequent PVCs in asymptomatic } \\
\text { patients with normal LV function }\end{array}$ & $\begin{array}{l}\text { Asymptomatic high-prevalence PVCs patients should } \\
\text { be monitored for progressive LVSD during a long- } \\
\text { term observation }\end{array}$ \\
\hline $\begin{array}{l}\text { Mountantonakis } \\
{[56]}\end{array}$ & 2011 & 69 & 62 & $51 \pm 16$ & $\begin{array}{l}\text { PICM with and without LV } \\
\text { dysfunction }\end{array}$ & $\begin{array}{c}\text { To examine the safety, efficacy, } \\
\text { and long-term effect of RFA on LV } \\
\text { function }\end{array}$ & $\begin{array}{l}\text { Frequent outflow tract PVCs are associated with CM } \\
\text { regardless of ventricle of origin. Significant }(>80 \%) \\
\text { reduction in PVCs improves LV function }\end{array}$ \\
\hline Yokokawa [57] & 2012 & 241 & 48 & $48 \pm 14$ & $\begin{array}{l}\text { Frequent PVCs with preserved } \\
\text { LVEF }(52 \pm 12) \text { referred for CA }\end{array}$ & $\begin{array}{l}\text { To assess determinants of PVC- } \\
\text { induced LV dysfunction }\end{array}$ & $\begin{array}{l}\text { The duration of palpitations and the absence of } \\
\text { symptoms are independently associated with PICM }\end{array}$ \\
\hline Penela [58] & 2013 & 80 & 58 & $53 \pm 12$ & $\begin{array}{l}\text { Patients with frequent PVC and } \\
\text { LV dysfunction with/out SHD }\end{array}$ & $\begin{array}{c}\text { To assess ablation benefit in } \\
\text { patients with frequent PVC and LV } \\
\text { dysfunction with/out SHD }\end{array}$ & $\begin{array}{l}\text { RFA in patients with depressed LVEF induced a } \\
\text { progressive clinical and functional improvement } \\
\text { related to baseline PVC burden }\end{array}$ \\
\hline Basiouny [59] & 2014 & 16 & 31 & $35 \pm 13$ & $\begin{array}{l}\text { Symptomatic RVOT-PVCs with } \\
\text { LV dysfunction with/out overt } \\
\text { SHD }\end{array}$ & $\begin{array}{c}\text { To evaluate the effect of RFA on } \\
\text { cardiac function in LV dysfunction } \\
\text { patients }\end{array}$ & $\begin{array}{l}\text { RFCA of frequent RVOT-PVC has a beneficial effect } \\
\text { on cardiac function in patients with depressed LVEF }\end{array}$ \\
\hline El Kadri [19] & 2015 & 30 & 60 & $59 \pm 12$ & Patients with NICM and PVCs & $\begin{array}{c}\text { To investigate the impact of } \\
\text { frequent PVCs on NICM patients }\end{array}$ & $\begin{array}{l}\text { In patients with frequent PVCs and NICM, PVC } \\
\text { ablation improves LVEF and functional class }\end{array}$ \\
\hline Latchamsetty [45] & 2015 & 1185 & 45 & $52 \pm 15$ & $\begin{array}{l}\text { Patients who underwent RFCA } \\
\text { for frequent idiopathic PVCs }\end{array}$ & $\begin{array}{l}\text { To assess outcomes and } \\
\text { complications of RFA and the } \\
\text { predictors of its efficacy }\end{array}$ & $\begin{array}{c}\text { RFA a low-risk and often effective treatment strategy } \\
\text { to eliminate PVCs and restore cardiac function in } \\
\text { PICM }\end{array}$ \\
\hline Blaye-Felice [48] & 2016 & 168 & 62 & $55 \pm 15$ & $\begin{array}{c}\text { Patients with PICM (LVEF } \\
<50 \% \text { ) and increased LV } \\
\text { dimensions due to frequent PVCs }\end{array}$ & $\begin{array}{l}\text { To test the correlation of various } \\
\text { factors to the presence PICM }\end{array}$ & $\begin{array}{l}\text { Lack of palpitations, PVC burden, and epicardial } \\
\text { origin are independent factors identifying patients } \\
\text { prone to developing PICM }\end{array}$ \\
\hline $\begin{array}{l}\text { Wojdyła- } \\
\text { Hordyńska [60] }\end{array}$ & 2017 & 109 & 56 & $55 \pm 17$ & $\begin{array}{l}\text { Patients referred to CA of } \\
\text { symptomatic frequent PVCs } \\
\text { refractory to medical therapy } \\
\text { with LVSD }\end{array}$ & $\begin{array}{l}\text { To assess the effect of CA and } \\
\text { PVC burden in patients with/out } \\
\text { SHD }\end{array}$ & $\begin{array}{l}\text { RFA improves LV function within six months in } \\
\text { patients with PVC burden }<20,000 / 24 \mathrm{~h}\end{array}$ \\
\hline Abdelhamid [61] & 2018 & 77 & 58 & $43 \pm 16$ & $\begin{array}{l}\text { Patients with LVSD }(\text { LVEF }< \\
50 \%) \text { and PVC burden }(>10 \%) \\
\text { refractory to medical treatment } \\
\text { with/out cv SHD }\end{array}$ & $\begin{array}{l}\text { To examine the effect of RFA } \\
\text { of monomorphic PVCs on LV } \\
\text { function recovery in patients with } \\
\text { LVSD with/out SHD }\end{array}$ & $\begin{array}{l}\text { PVCs elimination by RFA results in significant } \\
\text { improvement even restoration of LV function } \\
\text { regardless of PVC origin, or the presence of } \\
\text { concomitant SHD. PVCs burden pre-/post-ablation } \\
\text { are the main predictors of LVEF recovery }\end{array}$ \\
\hline
\end{tabular}


Albakri A (2019) Premature ventricular complexes (PVC)-induced cardiomyopathy: A review and meta-analysis of pathophysiology, diagnosis and clinical management

Other supporting diagnostic features included the presence of PVC morphology and axis on 12-lead ECG revealing bundle branch block, presence of outflow tract or facular morphology. However, the reversal of LV dysfunction following PVC suppression and a recovery of LV function with ablation confirms diagnosis of PICM. The most common site of PVCs origin reported in five studies was RVOT (37\%) followed by LVOT $(27 \%)[48,57,58,60,61]$.

The primary treatment for PICM in the 11 studies was radiofrequency ablation (RFA) for PVC that were refractory to medical therapy. Pooled analysis of data from five studies [19,48,57,59,61] revealed RFA therapy led to a significant reduction in the PVC burden from the baseline value of $26.09 \%$ (95\% CI: $24.69-27.50$ ) to post-RFA value of $0.89 \%$ (95\% CI: 0.72 to 1.07 ). The weighted mean difference (WMD) was $-22.31 \%(95 \%$ CI: -23.93 to -20.69 ; $\mathrm{p}=0.000)$ Figure 1. In eight studies [19-21,48,57-59,61], the drop in PVC-burden corresponded with a decrease in LVEF baseline (52.24\%; 95\% CI: 51.54-52.95) to post-RFA (63.19\%; 95\% CI: 62.46-63.92). The WMD between post-RFA and baseline LVEF was significant (WMD: $10.33 \%$; 95\% CI: 5.57 to $15.08 ; \mathrm{p}=0.000$; Figure 2). Pooled analysis of data from six studies [19-21,48,59,61] showed a significant reduction in LV end diastolic dimension (LVEDD) between baseline and postRFA therapy (WMD: $-4.52 \mathrm{~mm}$; 95\% CI: -6.72 to $-2.33 ; \mathrm{p}=0.000$; Figure 3 ) and LV end systolic dimension (LVESD: WMD: $-5.55 ; 95 \%$ CI: -7.40 to $-3.70 ; \mathrm{p}=0.000$; Figure 4 ) from data from three studies $[15,59,61]$.

Finally, factors predicting PICM were analysed. PVC-burden at baseline was associated with an increased risk of developing PICM (Odds Ratio [OR]: 1.050; 95\% CI: 1.031 to 1.069; $\mathrm{p}=0.000$; Figure 5) with data pooled from four studies $[45,48,57,58]$. The male sex also was associated with a significantly increased risk of developing PICM (OR: 2.20 ; 95\% CI; 1.56-3.1; $\mathrm{p}=0.000$; Figure 6 ) from data pooled from three studies $[45,48,58]$. Individual studies also reported other significant predictors of the development of PICM by multivariate analysis. These included asymptomatic status (OR: $13.1 ; 95 \% \mathrm{CI} ; 4.1$ to $37.8 ; \mathrm{p}=0.001$ ), long-standing palpitation duration (> 60 months) (OR: 20.1; 95\% CI: 6.3 to $64.1 ; \mathrm{p}=0.001$ ), QRS duration (OR: $0.99 ; 95 \% \mathrm{CI}: 0.96$ to $1.01 ; \mathrm{p}$ $=0.33$ ) [57], and symptom duration (OR: $1.09 ; 95 \%$ CI: 0.16 to $2.09 ; \mathrm{p}$ $=0.05)[61]$.

\section{Weighted Difference in Means and 95\% Cl: Pre- and Post-Ablation PVC Burden}

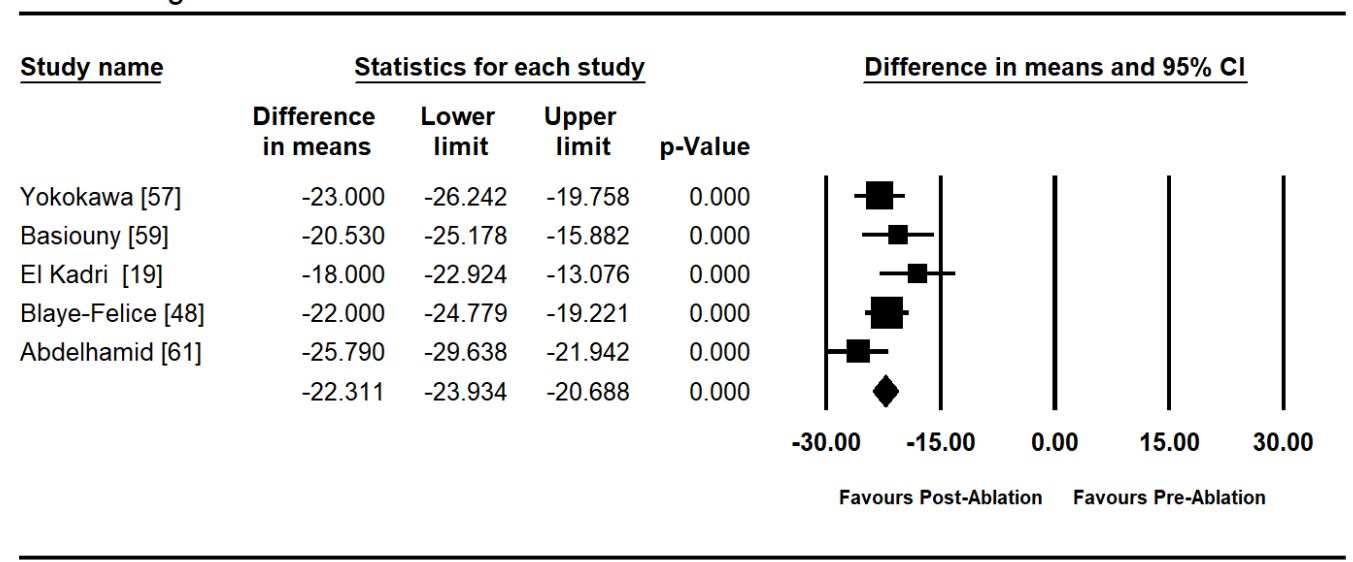

Heterogeinity: $Q=6.88 ; \mathrm{df}(Q)=4(p=0.143) ;$ Inconsistency $(I-S q u a r e d)=41.79 \%$

Figure 1. Forest plot of mean change between pre- and post-ablation PVC burden

Weighted Difference in Means and 95\% Cl: Pre- and Post-Ablation LVEF

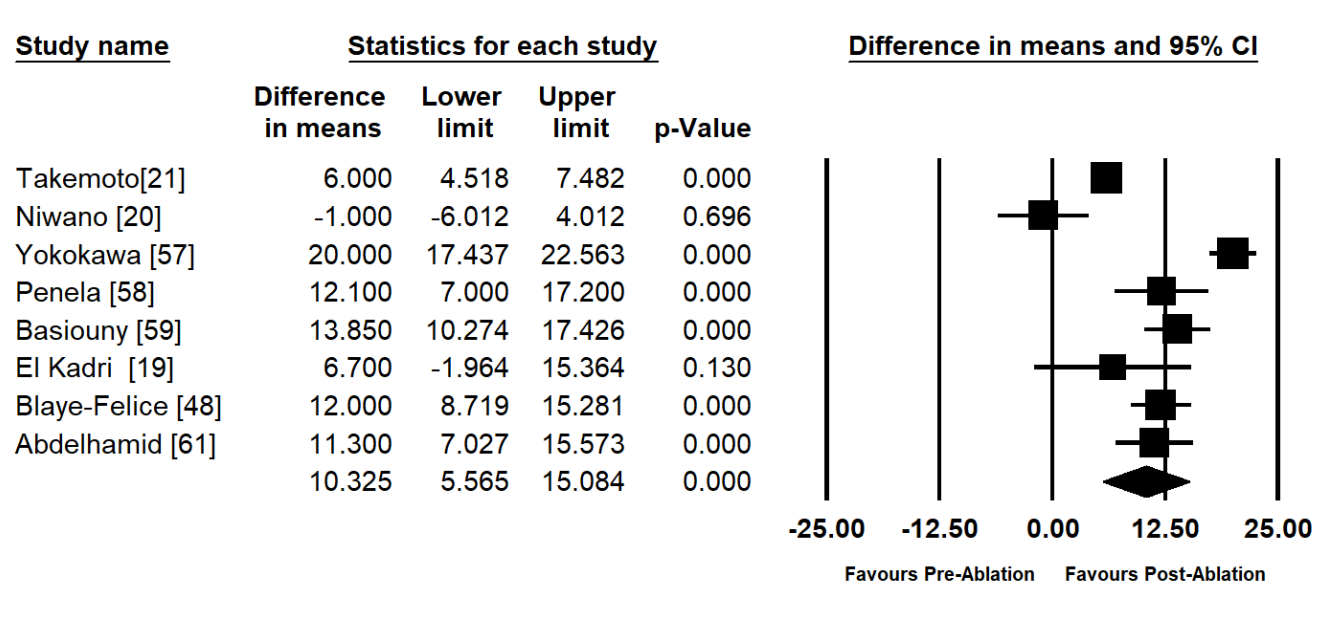

Heterogeinity: $Q=112.33 ; \mathrm{df}=7(p=0.000)$; Inconsistency $(I-S q u a r e d)=93.77 \%$

Figure 2. Forest plot of mean change between pre- and post-ablation LVEF 


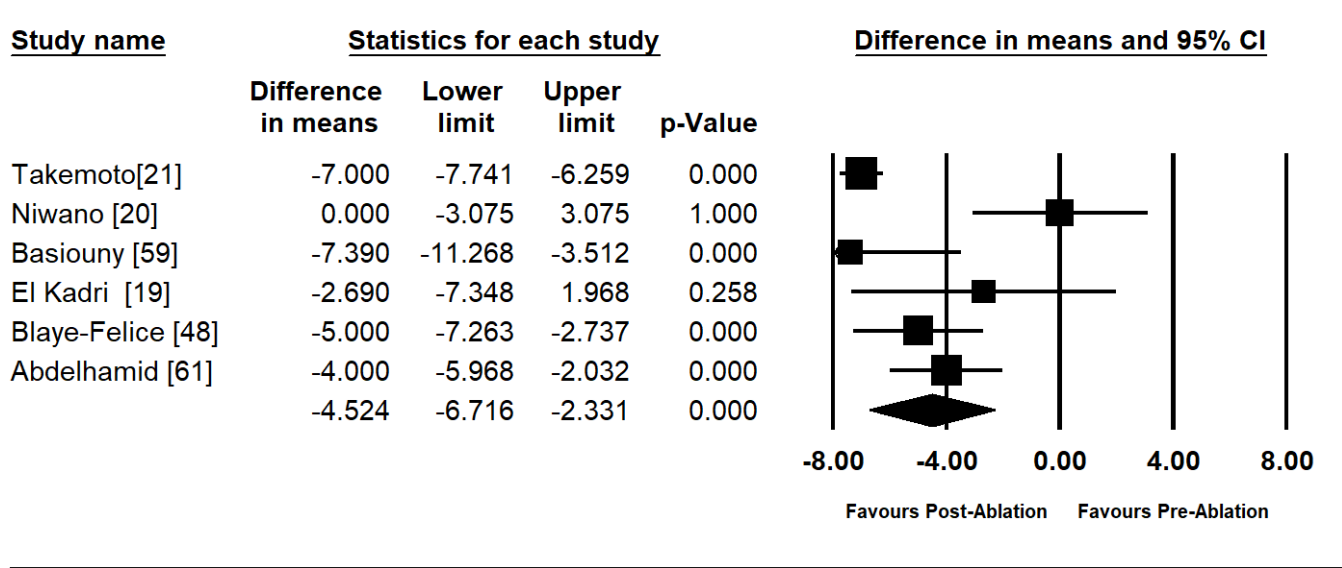

Heterogeinity: $Q=28.51 ; \mathrm{df}(Q)=5(p=0.000)$; Inconsistency $(I-S q u a r e d)=82.46 \%$

Figure 3. Forest plot of mean change between pre- and post-ablation LVEDD

Weighted Difference in Means and 95\% Cl: Pre- and Post-Ablation LVESD

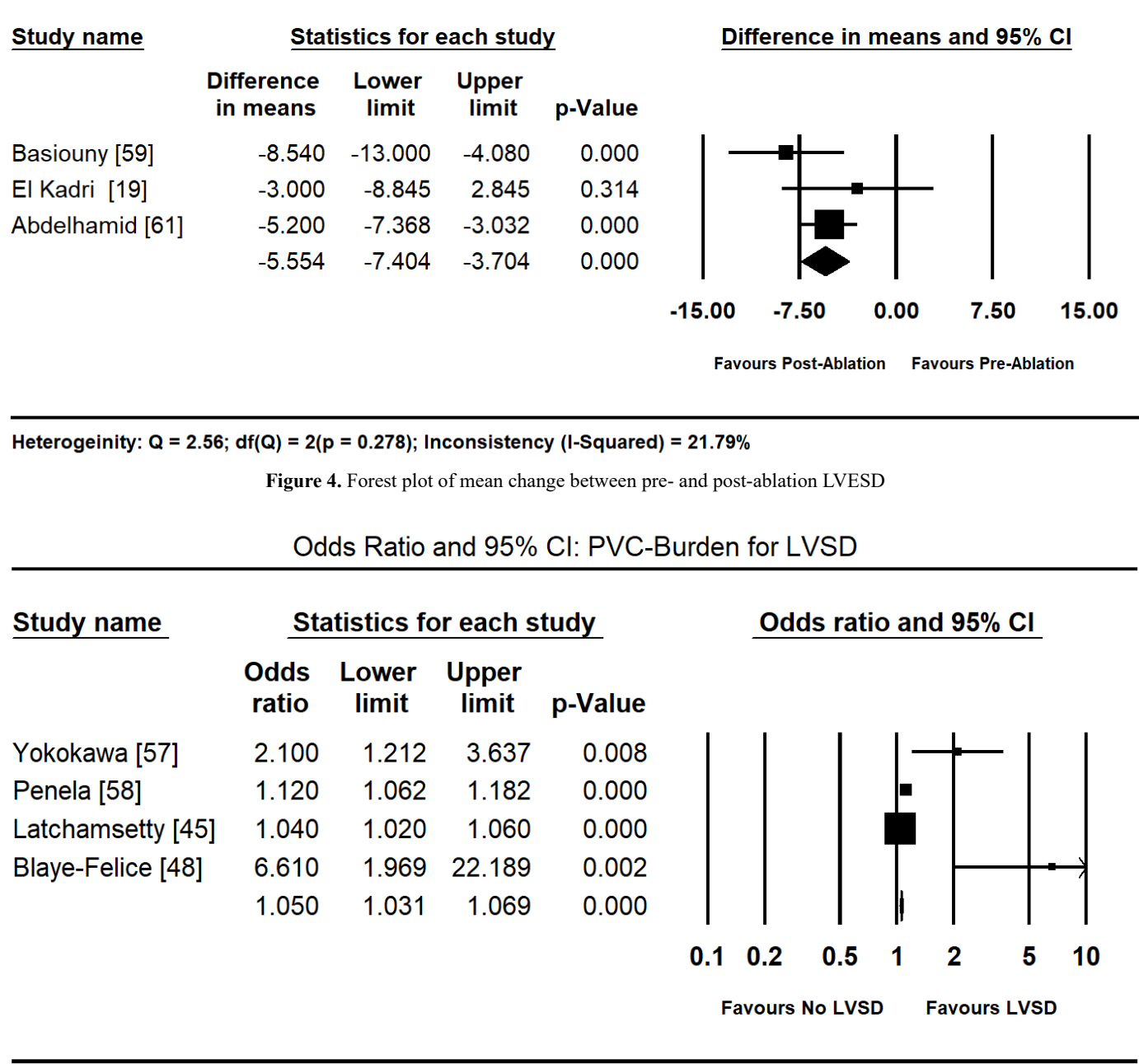

Heterogeinity: $Q=21.50 ; \operatorname{df}(Q)=3(p=0.000) ;$ Inconsistency $(I-S q u a r e d)=86.04 \%$

Figure 5. Forest plot of odds ratio for PICM comparing PVC-burden 


\begin{tabular}{lcccc} 
Study name & & \multicolumn{5}{c}{ Statistics for each study } \\
& $\begin{array}{c}\text { Odds } \\
\text { ratio }\end{array}$ & $\begin{array}{c}\text { Lower } \\
\text { limit }\end{array}$ & $\begin{array}{c}\text { Upper } \\
\text { limit }\end{array}$ & p-Value \\
Penela [58] & 3.330 & 1.266 & 8.757 & 0.015 \\
Latchamsetty [45] & 1.660 & 1.064 & 2.590 & 0.026 \\
Blaye-Felice [48] & 3.360 & 1.753 & 6.441 & 0.000 \\
& 2.205 & 1.564 & 3.108 & 0.000
\end{tabular}

\section{Odds ratio and $95 \% \mathrm{Cl}$}

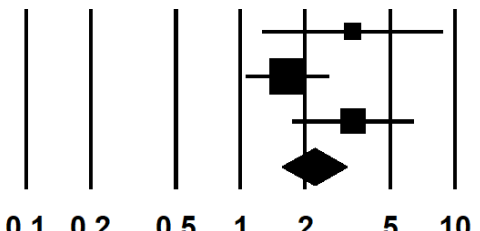

$\begin{array}{lllllll}0.1 & 0.2 & 0.5 & 1 & 2 & 5 & 10\end{array}$

Favours No LVSD Favours LVSD

Heterogeinity: $Q=3.87 ; \mathrm{df}(\mathrm{Q})=2(p=0.144)$; Inconsistency $(\mathrm{I}-$ Squared $)=48.37 \%$

Figure 6. Forest plot of odds ratio for PICM comparing sex

\section{Discussion}

There is no single confirmatory diagnostic test for PICM. It is a diagnosis per exclusionem (diagnosis of exclusion). Diagnostic workup rests on demonstrating a combination of high PVC burden $(>10 \%)$ or frequency $>10,000$ beats per day on 24 hour Holter monitoring, severe LV systolic dysfunction, the presence of PVC morphology (bundle branch block) and reversal of LV dysfunction after therapeutic suppression of PVC. Reversal here means improvement of LEVF by $>15 \%$ or normalization of the LVEF after effective ablation therapy. The primary treatment target of PICM is the suppression of PVCs. In the present meta-analysis, RFA is a common and efficacious therapy for PICM patients. RFA leads to a significant suppression of mean PVC burden from $>20 \%$ to $<1 \%$ accompanied by a marked improvement in LV systolic function both in patients with or without LV dysfunction. Mean LVEF increased by $10.3 \%$ accompanied by a significant decrease in LVEDD and LVESD by $4.52 \mathrm{~mm}$ and $5.55 \mathrm{~mm}$ respectively. Patients with a high PVC-burden and male sex are more likely to develop PICM. Other predicting factors for PICM mentioned in individual studies are asymptomatic status, long-standing palpitation, QRS duration and symptom duration.

\section{Diagnosis workup}

The present findings on the utility of PVC burden and morphology, and LV dysfunction to support the diagnosis of PICM is consistent with present review and guidelines $[26,62,63]$. The onset of frequent PVCs relative to the onset of LV dysfunction remains unknown [64]. It is vital to determine the primary disorder because PICM is a potentially reversible myocardial disease $[65,66]$. Clinical signs and symptoms of PICM are either non-specific or non-existent (in asymptomatic patients). Patients can present with debilitating symptoms (palpitations) or other symptoms such as chest pain, presyncope, syncope or HF manifested with decreased effort tolerance [67]. A greater majority of patients with PVCs are healthy with no known or demonstrable heart disease. A detailed family history may be helpful to exclude familial DCM. Findings of physical examination is often normal except irregular heart rhythms when PVCs are frequent. However, physical examination is useful to assess signs of an underlying structural heart disease and HF such as an elevated jugular venous pressure, abnormal cardiac sounds, pulmonary rales, abnormal arterial pulses to peripheral oedema. Heart murmurs should raise suspicion of valvular or congenital heart disease when presenting in a young patient [63].
The initial workup in patients presenting with frequent PVCs is to detect and quantify PVCs through monitoring cardiac rhythm using 12lead ECG and ambulatory Holter monitoring. The 12-lead ECG detects the presence and morphology of PVCs. Holter monitoring provides a continuous recording usually in at least two or three leads. A patient wears the monitor underneath clothing and returns it for download of the collected data on sinus rhythm. Ambulatory Holter monitoring for at least 24 hours helps to estimate a patient's PVC burden (expressed as the percentage of heartbeats that were ventricular extrasystoles during the monitoring period). A single 24-hour recording may not reflect the true PVC load because of day-to-day variability. A strong suspicion that frequent PVCs may cause LV dysfunction may warrant extended Holter monitoring for 48 to 72 hours or several 24-hour recordings [68]. Holter monitoring can also identify the presence of any related VT as well as additional supraventricular arrhythmias or bradycardia during the monitoring period $[62,63]$.

Both ECG and Holter monitoring record only snapshots of the rhythm during the period when the patient is actually hooked up. However, many patients experience PVCs in clusters every few days or weeks. Such a pattern is unlikely to be detected by a single ECG or a 24 to 48 hour Holter monitoring [63]. In these scenarios, a 30-day ambulatory event monitor (also known as wearable loop recorder) becomes an important diagnostic tool. It uses the same concept as Holter monitoring but provides a continuous loop recording of cardiac rhythm digitally stored in clips after activation. Cardiac rhythm monitoring can also be dome by mobile outpatient cardiac telemetry - a wearable ambulatory device providing continuous recordings, realtime automatic detections and patient-activated symptom recording. It can be used up to six weeks. However, telemetry is rarely a part of a PVC evaluation although a cardiac electrophysiologist or a cardiologist can order its use [63].

The second step in the diagnosis of PICM is evaluation for LV cardiac structure and function by non-invasive cardiac imaging methods. Surface echocardiography is useful to detect and exclude potential known causes of frequent PVCs such as valvular pathology, regional wall motion abnormalities, or non-compaction. Standard echocardiography is also useful to detect cardiac features associated with PICM including decreased LVEF, increased systolic and diastolic dimensions, global wall motion abnormalities and mitral regurgitation [68]. Two dimensional (2D) speckle tracking-strain imaging (STI) can also detect altered LV contractility despite preserved LVEF $[69,70]$. 
However, whether 2D-STI can predict the reduction in LVEF in patients with high frequency of PVCs remains to be studied. Advanced cardiac imaging such as computed tomography, MRI or positron-emission tomography are reserved for specific clinical indications such as congenital heart diseases, suspected cardiac sarcoidosis, and infiltrative heart disease and for differential diagnosis for specific CMs [63]. Cardiac MRI is useful for the detection of arrhythmogenic RV cardiomyopathy with LV involvement and infiltrative disease when clinically suspected. Coronary angiography is a useful test for every patient with reduced LV systolic function to exclude significant coronary artery disease (CAD) except for patients with a low cardiovascular risk. Appropriate workup may be warranted to exclude other causes of $\mathrm{CM}$ including those related to drugs or toxins, infectious diseases and endocrinopathies.

It should be noted that PICM is a diagnosis per exclusionem, the underlying structural disease causing frequent PVCs must be ruled out. In addition, given that PVCs can be a cause and a consequence of PICM, it may be difficult to isolate the primary disorder [66,71]. In many patients, the onset of frequent PVCs relative to the onset of LV dysfunction is not known [64]. It is vital to identify the primary disorder because of the potential reversibility of PICM $[65,66]$.

\section{Therapeutic options}

The present meta-analysis finds RFA of PVCs is an efficacious therapy for PICM patients in the reduction of PVC burden and improvement in LV function. The findings are consistent with consensus guidelines for the treatment of PICM. The AHA scientific statement for the diagnosis and treatment of specific forms of DCM recommends RFA in PICM patients [9]. The statement supports the findings that RFA reduces PVC burden in patients with LV dysfunction, improving both LV function in both structurally normal and abnormal hearts. In patients with LV dysfunction, RFA improves LV dilatation (reduces LV systolic and diastolic dimensions) mitral regurgitation and ejection fraction [60]. Reasons for indications for treatment by ablation are LV dysfunction and frequent PVCs $(10,000$ to 20,000 or $>10 \%$ of total beats over 24 hour period) if the clinical suspicion for PICM is high. The patient is usually followed for $>3$ to 24 months to evaluate post-treatment PVC frequency, and LV size and function.

Generally, PICM treatment includes the management of secondary causes, pharmacotherapy to suppress PVCs or RFA to reduce or eliminate PVCs. Reducing or stopping caffeine and alcohol intake, and having a better control of emotional stress have modest effectiveness in reducing PVC frequency [72,73]. Presently, evidence supporting any treatment for asymptomatic patients with frequent PVCs is lacking although beta-blockers or calcium channel blockers, if tolerated, could be discussed [3]. In such patients, regular assessment of their LVEF is recommended and patients advised to report to hospital if they develop HF symptoms. A high PVC burden is a leading predictor for developing $\mathrm{CM}$ but the majority of patients presenting with frequent PVCs have preserved LVEF and may not develop CM suggesting a differential susceptibility among individuals despite having a similar PVC burden $[17,20]$. The suppression of PVCs can be through pharmacotherapy or RFA.

Pharmacotherapy is one of the clinical approaches used to suppress PVCs. In the presence of symptoms, medication such as beta-blocker or non-dihydropyridine calcium channel blockers may be the first-line therapy [74]. However, in patients presenting with frequent PVCs and a preserved LVEF, the preferred treatment is reassurance and counselling of the patient, with close follow-up if the patient is asymptomatic. Usually, long-term prognosis of frequent PVCs is benign in a majority of patients presenting with frequent PVCs and preserved LVEF $[3,26]$. Previous studies have reported atenolol significantly reduced symptom frequency and PVC burden and antiarrhythmia (flecainide or sotalol) may be considered when the patients is non-responsive to beta-blockers or calcium channel blockers [75]. Patients taking flecainide (91\%) and mexiletine (55\%) had a PVC reduction of $>70 \%$ [76]. However, in patients with overt LV dysfunction, Class IA or IC drugs such as flecainide or propafenone are not recommended because of their side effect profiles especially the propensity for pro-arrhythmic effects and adverse impact on survival [77]. Amiodarone has also been shown to suppress PVC and improve LV function in patients with HF and asymptomatic ventricular arrhythmias [55,78,79].

Besides pharmacotherapy, RFA has been shown to be effective in reducing or even eliminating PVCs. It is becoming a first-line treatment for patients presenting with RVOT PVCs because of the high success rate of RFA and low risk of complications. With the support of intracardiac echocardiography and electro-anatomical mapping systems, RFA is also safe in other formerly riskier locations such as the aortic root or papillary muscle [3].

\section{Conclusion}

There is increasing recognition that frequent PVCs are reversible cause of CM characterized by LV systolic dysfunction. PVCs are common in patients with an underlying structural heart disease associated with a poor prognosis as well as in asymptomatic patients considered a benign entity with no prognostic implications. Independent predictors of the development of PICM are a high PVC burden $>10,000$ beats per 24 hours or $>10 \%$, PVC origin, PVC QRS features, interpolation and coupling intervals, PVC duration, circadian variability and the male sex. The underlying pathophysiologic mechanisms are not clear although tachycardia-induced mechanism and electromechanical dyssynchrony have been described. PICM patients may present as asymptomatic, symptomatic acutely from individual PVCs or symptomatic chronic due to cumulative effects of PVCs. Diagnosis of PICM is by exclusion. Demonstration of a high PVC burden, LV systolic dysfunction and reversible LV dysfunction with ablation in the absence of other known causes of LV dysfunction confirms PICM. The mainstay therapy of PICM is PVC suppression through either pharmacotherapy or RFA. Effective RFA therapy reduces the PVC burden and improves LV function both in patients with structurally abnormal or normal hearts. However, treatment for asymptomatic patients with frequent PVCs is not clear although it is prudent to monitor LV function periodically due to the potential for deterioration of LV function during long-term follow-up.

\section{References}

1. Kennedy HL, Whitlock JA, Sprague MK, Kennedy LJ, Buckingham TA et al. (1985) Long-term follow-up of asymptomatic healthy subjects with frequent and complex ventricular ectopy. $N$ Engl J Med 312: 193-197. [Crossref]

2. Gaita F, Giustetto C, Di Donna P, Richiardi E, Libero L et al. (2001) Long-term followup of right ventricular monomorphic extrasystoles. $J$ Am Coll Cardiol 38: 364-370. [Crossref]

3. Panizo JG, Barra S, Mellor G, Heck P, Agarwal S (2018) Premature ventricular complex-induced cardiomyopathy. Arrhythm Electrophysiol Rev 7: 128-134. [Crossref]

4. Chugh SS, Shen WK, Luria DM, Smith HC (2000) First evidence of premature ventricular complex-induced cardiomyopathy: a potentially reversible cause of heart failure. J Cardiovasc Electrophysiol 11: 328-329. [Crossref]

5. Efremidis M, Letsas KP, Sideris A, Kardaras F (2008) Reversal of premature ventricular complex-induced cardiomyopathy following successful radiofrequency catheter ablation. Europace 10: 769-770. [Crossref] 
Albakri A (2019) Premature ventricular complexes (PVC)-induced cardiomyopathy: A review and meta-analysis of pathophysiology, diagnosis and clinical management

6. Shiraishi H, Ishibashi K, Urao N, Tsukamoto M, Hyogo M et al. (2002) A case of cardiomyopathy induced by premature ventricular complexes. Circ J 66: 1065-1067. [Crossref]

7. Duffee DF, Shen WK, Smith HC (1998) Suppression of frequent premature ventricular contractions and improvement of left ventricular function in patients with presumed idiopathic dilated cardiomyopathy. Mayo Clin Proc 73: 430-433. [Crossref]

8. Huizar JF, Kaszala K, Potfay J, Minisi AJ, Lesnefsky EJ et al. (2011) Left ventricular systolic dysfunction induced by ventricular ectopy: a novel model for premature ventricular contraction-induced cardiomyopathy. Circ Arrhythm Electrophysiol 4: 543549. [Crossref]

9. Bozkurt B, Colvin M, Cook J, Cooper LT, Deswal A et al. (2016) Current diagnostic and treatment strategies for specific dilated cardiomyopathies: a scientific statement from the American Heart Association. Circulation 134: e579-e646. [Crossref]

10. Pinto YM, Elliott PM, Arbustini E, Adler Y, Anastasakis A et al. (2016) Proposal for a revised definition of dilated cardiomyopathy, hypokinetic non-dilated cardiomyopathy, and its implications for clinical practice: a position statement of the ESC working group on myocardial and pericardial diseases. Eur Heart J 37: 1850-1858. [Crossref]

11. Kennedy HL, Whitlock JA, Sprague MK, Kennedy LJ, Buckingham TA, Goldberg RJ (1985) Long-term follow-up of asymptomatic healthy subjects with frequent and complex ventricular ectopy. $N$ Engl J Med 312: 193-197. [Crossref]

12. Ng GA (2006). Treating patients with ventricular ectopic beats. Heart 92: 1707-1712. [Crossref]

13. Messineo FC (1989) Ventricular ectopic activity: prevalence and risk. Am J Cardiol 64: 53J-56J. [Crossref]

14. Southall DP, Johnston F, Shinebourne EA, Johnston PG (1981) 24-hour electrocardiographic study of heart rate and rhythm patterns in population of healthy children. Br Heart J 45: 281-291. [Crossref]

15. Camm AJ, Evans KE, Ward DE, Martin A (1980) The rhythm of the heart in active elderly subjects. Am Heart J 99: 598-603. [Crossref]

16. Latchamsetty R, Bogun F (2016) Premature ventricular complex-induced cardiomyopathy. Rev Esp Cardiol (Engl Ed) 69: 365-369. [Crossref]

17. Yarlagadda RK, Iwai S, Stein KM, Markowitz SM, Shah BK et al. (2005) Reversal of cardiomyopathy in patients with repetitive monomorphic ventricular ectopy originating from the right ventricular outflow tract. Circulation 112: 1092-1097. [Crossref]

18. Baman TS, Lange DC, Ilg KJ, Gupta SK, Liu TY et al. (2010) Relationship between burden of premature ventricular complexes and left ventricular function. Heart Rhythm 7: 865-869. [Crossref]

19. El Kadri M, Yokokawa M, Labounty T, Mueller G, Crawford T et al. (2015) Effect of ablation of frequent premature ventricular complexes on left ventricular function in patients with non-ischemic cardiomyopathy. Heart Rhythm 12: 706-713. [Crossref]

20. Niwano S, Wakisaka Y, Niwano H, Fukaya H, Kurokawa S et al. (2009) Prognostic significance of frequent premature ventricular contractions originating from the ventricular outflow tract in patients with normal left ventricular function. Heart 95: 1230-1237. [Crossref]

21. Takemoto M, Yoshimura H, Ohba Y, Matsumoto Y, Yamamoto U et al. (2005) Radiofrequency catheter ablation of premature ventricular complexes from right ventricular outflow tract improves left ventricular dilation and clinical status in patients without structural heart disease. J Am Coll Cardiol 45: 1259-1265. [Crossref]

22. Bogun F, Crawford T, Reich S, Koelling TM, Armstrong W et al. (2007) Radiofrequency ablation of frequent, idiopathic premature ventricular complexes: comparison with a control group without intervention. Heart Rhythm 4: 863-867. [Crossref]

23. Taieb JM, Maury P, Shah D, Duparc A, Galinier M et al. (2007) Reversal of dilated cardiomyopathy by the elimination of frequent left or right premature ventricular contractions. J Interv Card Electrophysiol 20: 9-13. [Crossref]

24. Kanei Y, Friedman M, Ogawa N, Hanon S, Lam P et al. (2008) Frequent premature ventricular complexes originating from the right ventricular outflow tract are associated with left ventricular dysfunction. Ann Noninvasive Electrocardiol 13: 81-85. [Crossref]

25. Hasdemir C, Ulucan C, Yavuzgil O, Yuksel A, Kartal Y et al. (2011) Tachycardiainduced cardiomyopathy in patients with idiopathic ventricular arrhythmias: the incidence, clinical and electrophysiologic characteristics, and the predictors. $J$ Cardiovasc Electrophysiol 22: 663-668. [Crossref]

26. Cha YM, Lee GK, Klarich KW, Grogan M (2012) Premature ventricular contractioninduced cardiomyopathy: a treatable condition. Circulation: Circ Arrhythm Electrophysiol 5: 229-236. [Crossref]
27. Ban JE, Kim YH (2013) PVC-induced cardiomyopathy: the cut-off value for the premature ventricular complex burden. Europace 15: 1063-1064. [Crossref]

28. Ban JE, Park HC, Park JS, Nagamoto Y, Choi JI et al. (2012) Electrocardiographic and electrophysiological characteristics of premature ventricular complexes associated with left ventricular dysfunction in patients without structural heart disease. Europace 15 : 735-741. [Crossref]

29. Gami AS, Noheria A, Lachman N, Edwards WD, Friedman PA et al. (2011) Anatomical correlates relevant to ablation above the semilunar valves for the cardiac electrophysiologist: a study of 603 hearts. J Interv Card Electrophysiol 30: 5-15. [Crossref]

30. Del Carpio MF, Syed FF, Noheria A, et al. (2011) Characteristics of premature ventricular complexes as correlates of reduced left ventricular systolic function: study of the burden, duration, coupling interval, morphology and site of origin of PVCs. $J$ Cardiovasc Electrophysiol 22: 791-798. [Crossref]

31. Steinberg JS, Fischer A, Wang P, Schuger C, Daubert J et al. (2005) The clinica implications of cumulative right ventricular pacing in the multicentre automatic defibrillator trial II. J Cardiovasc Electrophysiol. 2005;16: 359-365. [Crossref]

32. Blanc JJ, Fatemi M, Bertault V, Baraket F, Etienne Y (2005) Evaluation of left bundle branch block as a reversible cause of non-ischaemic dilated cardiomyopathy with severe heart failure. A new concept of left ventricular dyssynchrony-induced cardiomyopathy. Europace 7: 604-610. [Crossref]

33. Gardiwal A, Yu H, Oswald H, Luesebrink U, Ludwig A et al. (2008) Right ventricular pacing is an independent predictor for ventricular tachycardia/ventricular fibrillation occurrence and heart failure events in patients with an implantable cardioverterdefibrillator. Europace 10: 358-363. [Crossref]

34. Lee SJ, McCulloch C, Mangat I, Foster E, De Marco T et al. (2003) Isolated bundle branch block and left ventricular dysfunction. J Card Fail 9: 87-92. [Crossref]

35. Spragg DD, Kass DA (2006) Pathobiology of left ventricular dyssynchrony and resynchronization. Prog Cardiovasc Dis 49: 26-41. [Crossref]

36. Tantengco MV, Thomas RL, Karpawich PP (2001) Left ventricular dysfunction after long-term right ventricular apical pacing in the young. J Am Coll Cardiol 37: 2093 2100. [Crossref]

37. Yokokawa M, Kim HM, Good E, Crawford T, Chugh A et al. (2012) Impact of QRS duration of frequent premature ventricular complexes on the development of cardiomyopathy. Heart Rhythm 9: 1460-1464. [Crossref]

38. Moulton KP, Medcalf T, Lazzara R (1990) Premature ventricular complex morphology A marker for left ventricular structure and function. Circulation 81: 1245-1251. [Crossref]

39. Olgun H, Yokokawa M, Baman T, Kim HM, Armstrong W et al. (2011) The role of interpolationin PVC-induced cardiomyopathy. Heart Rhythm 8: 1046-1049. [Crossref]

40. Sun Y, Blom NA, Yu Y, Ma P, Wang Y et al. (2003) The influence of premature ventricular contractions on left ventricular function in asymptomatic children without structural heart disease: an echocardiographic evaluation. Int J Cardiovasc Imaging 19: 295-299. [Crossref]

41. Otsuji Y, Toda H, Kisanuki A, Koyano T, Kuroiwa R et al. (1994) Influence of left ventricular filling profile during preceding control beats on pulse pressure during ventricular premature contractions. Eur Heart J 15: 462-467. [Crossref]

42. Bradfield JS, Homsi M, Shivkumar K, Miller JM (2014) Coupling interval variability differentiates ventricular ectopic complexes arising in the aortic sinus of valsalva and great cardiac vein from other sources: mechanistic and arrhythmic risk implications. $J$ Am Coll Cardiol 63: 2151-2158. [Crossref]

43. Bas HD, Baser K, Hoyt J, Yokokawa M, LaBounty T et al. (2015) Effect of circadian variability in frequency of premature ventricular complexes on left ventricular function. Heart Rhythm 13: 98-102. [Crossref]

44. Hamon D, Abehsira G, Gu K, Liu A, Sadron MB et al. (2018) Circadian variability patterns predict and guide premature ventricular contraction ablation procedural inducibility and outcomes. Heart Rhythm 15: 99-106. [Crossref]

45. Latchamsetty R, Yokokawa M, Morady F, Kim HM, Mathew S et al. (2015) Multicenter outcomes for catheter ablation of idiopathic premature ventricular complexes. JACC: Clin Electrophysiol 1: 116-123. [Crossref]

46. Sirichand S, Killu AM, Padmanabhan D, Hodge DO, Chamberlain AM et al. (2017) Incidence of idiopathic ventricular arrhythmias: a population-based study. Circ Arrhythm Electrophysiol 10: e004662. [Crossref] 
47. Humphries KH, Kerr CR, Connolly SJ, Klein G, Boone JA et al. (2001) New-onset atrial fibrillation: sex differences in presentation, treatment, and outcome. Circulation 103: 2365-2370. [Crossref]

48. Blaye-Felice MS, Hamon D, Sacher F, Pascale P, Rollin A et al. (2016) Premature ventricular contraction-induced cardiomyopathy: related clinical and electrophysiologic parameters. Heart Rhythm 13: 103-110. [Crossref]

49. Gwag HB, Kim EK, Hwang JK, Park SJ, On YK et al. (2018) Is the stroke volume during post-ectopic beat associated with ventricular premature complex-related symptoms? Europace 20: f204-f210. [Crossref]

50. Saurav A, Smer A, Abuzaid A, Bansal O, Abuissa H (2015) Premature Ventricular Contraction-Induced Cardiomyopathy. Clin Cardiol 38: 251-258. [Crossref]

51. Mukherjee R, Hewett KW, Spinale FG (1995) Myocyte electrophysiological properties following the development of supraventricular tachycardia-induced cardiomyopathy. $J$ Mol Cell Cardiol 27: 1333-1348. [Crossref]

52. Xiao L, Coutu P, Villeneuve LR, Tadevosyan A, Maguy A et al. (2008) Mechanisms underlying rate-dependent remodelling of transient outward potassium current in canine ventricular myocytes. Circ Res 103: 733-742. [Crossref]

53. Dandamudi G, Rampurwala AY, Mahenthiran J, Miller JM, Das MK (2008) Persistent left ventricular dilatation in tachycardia-induced cardiomyopathy patients after appropriate treatment and normalization of ejection fraction. Heart Rhythm 5: 11111114. [Crossref]

54. Pol LC, Deyell MW, Frankel DS, Benhayon D, Squara F et al. (2014) Ventricular premature depolarization QRS duration as a new marker of risk for the development of ventricular premature depolarization-induced cardiomyopathy. Heart Rhythm 11: 299-306. [Crossref]

55. Deyell MW, Park KM, Han Y, Frankel DS, Dixit S et al. (2012) Predictors of recovery of left ventricular dysfunction after ablation of frequent ventricular premature depolarizations. Heart Rhythm 9: 1465-1472. [Crossref]

56. Mountantonakis SE, Frankel DS, Gerstenfeld EP, Dixit S, Lin D et al. (2011) Reversa of outflow tract ventricular premature depolarization-induced cardiomyopathy with ablation: effect of residual arrhythmia burden and pre-existing cardiomyopathy on outcome. Heart rhythm 8: 1608-1614. [Crossref]

57. Yokokawa M, Kim HM, Good E, Chugh A, Pelosi Jr F et al. (2012) Relation of symptoms and symptom duration to premature ventricular complex-induced cardiomyopathy. Heart Rhythm 9: 92-95. [Crossref]

58. Penela D, Taxis CV, Aguinaga L, Fernández-Armenta J, Mont L et al. (2013) Neurohormonal, structural, and functional recovery pattern after premature ventricular complex ablation is independent of structural heart disease status in patients with depressed left ventricular ejection fraction: a prospective multi-centre study. $J$ Am Coll Cardiol 62: 1195-1202. [Crossref]

59. Basiouny T, Kholeif HA, El-Tahan MH, Karim M, Attia W et al. (2014) Radiofrequency catheter ablation of premature ventricular complexes from right ventricular outflow tract in patients with left ventricular dilation and/or dysfunction. Egyptian Heart Journal 66: 351-361.

60. Wojdyła-Hordyńska A, Kowalski O, Hordyński GJ, Dinov B, Sommer P et al. (2017) The effect of radiofrequency catheter ablation of frequent premature ventricular complexes and arrhythmia burden on left ventricular function. Kardiol Pol 75: 698704. [Crossref]

61. Abdelhamid MA, Samir R (2018) Reversal of premature ventricular complexes induced cardiomyopathy. Influence of concomitant structural heart disease. Indian Heart J 70: 410-415. [Crossref]
62. Noheria A, Deshmukh A, Asirvatham SJ (2015) Ablating premature ventricular complexes: justification, techniques, and outcomes. Methodist Debakey Cardiovasc J 11: 109. [Crossref]

63. Cantillon DJ (2013) Evaluation and management of premature ventricular complexes. Cleve Clin J Med 80: 377-387. [Crossref]

64. Wilber DJ (2009) Ventricular ectopic beats: not so benign. Heart 95: 1209-1210. [Crossref]

65. Tikkanen JT, Anttonen O, Junttila MJ, Aro AL, Kerola T et al. (2009) Long-term outcome associated with early repolarization on electrocardiography. $N$ Engl $J$ Med 361: 2529-2537. [Crossref]

66. Bhushan M, Asirvatham SJ (2009) The conundrum of ventricular arrhythmia and cardiomyopathy: which abnormality came first? Curr Heart Fail Rep 6: 7-13. [Crossref]

67. Sheldon SH, Gard JJ, Asirvatham SJ (2010) Premature ventricular contractions and nonsustained ventricular tachycardia: association with sudden cardiac death, risk stratification, and management strategies. Indian Pacing Electrophysiol J 10: 357-371. [Crossref]

68. Shanmugam N, Chua TP, Ward D (2006) 'Frequent' ventricular bigeminy: a reversible cause of dilated cardiomyopathy. How frequent is 'frequent'? Eur J Heart Fail 8: 869873. [Crossref]

69. Delgado V, Ypenburg C, van Bommel RJ, Tops LF, Mollema SA et al. (2008) Assessment of left ventricular dyssynchrony by speckle tracking strain imaging comparison between longitudinal, circumferential, and radial strain in cardiac resynchronization therapy. J Am Coll Cardiol 51: 1944-1952. [Crossref]

70. Wijnmaalen AP, Delgado V, Schalij MJ, van Huls van Taxis CF, Holman ER et al. (2010) Beneficial effects of catheter ablation on left ventricular and right ventricular function in patients with frequent premature ventricular contractions and preserved ejection fraction. Heart 96: 1275-1280. [Crossref]

71. Abdalla IS, Prineas RJ, Neaton JD, Jacobs DR Jr, Crow RS (1987) Relation between ventricular premature complexes and sudden cardiac death in apparently healthy men. Am J Cardiol 60: 1036-1042. [Crossref]

72. Dixit S, Stein PK, Dewland TA, Dukes JW, Vittinghoff E et al. (2016) Consumption of caffeinated products and cardiac ectopy. J Am Heart Assoc 5: e002503. [Crossref]

73. Prineas RJ, Jacobs DR Jr, Crow RS, Blackburn H (1980) Coffee, tea and VPB. $J$ Chronic Dis 33: 67-72. [Crossref]

74. Wellens HJ (1999) Catheter ablation of cardiac arrhythmias: usually cure, but complications may occur. Circulation 99: 195-197. [Crossref]

75. Bardy GH, Lee KL, Mark DB, Poole JE, Packer DL et al. (2005) Amiodarone or an implantable cardioverter-defibrillator for congestive heart failure. $N$ Engl J Med 352 225-237. [Crossref]

76. Martínez-Alanis M, Ruiz-Velasco S, Lerma C (2016) Quantitative analysis of ventricular ectopic beats in short-term RR interval recordings to predict imminent ventricular tachyarrhythmia. Int J Cardiol 225: 226-233. [Crossref]

77. Yokokawa M, Good E, Crawford T, Chugh A, Pelosi Jr F et al. (2013) Reasons for failed ablation for idiopathic right ventricular outflow tract-like ventricular arrhythmias. Heart Rhythm 10: 1101-1108. [Crossref]

78. McDonnell K, Rhee E,Srivathsan K, Su W (2014) Novel utility of cryoablation for ventricular arrhythmias arising from the left aortic cusp near the left main coronary artery: A case series. Heart Rhythm 11: 34-38. [Crossref]

79. Mountantonakis SE, Frankel DS, Gerstenfeld EP, Dixit S, Lin D et al. (2011) Reversa of outflow tract ventricular premature depolarization-induced cardiomyopathy with ablation: effect of residual arrhythmia burden and pre-existing cardiomyopathy on outcome. Heart Rhythm 8: 1608-1614. [Crossref]

Copyright: (C2019 Albakri A. This is an open-access article distributed under the terms of the Creative Commons Attribution License, which permits unrestricted use, distribution, and reproduction in any medium, provided the original author and source are credited. 